\section{Desenvolvimento de variômetro fluxgate}

Benyosef, L.C.C. \& Bochner, M. - Observatório Nacional, Brazil.

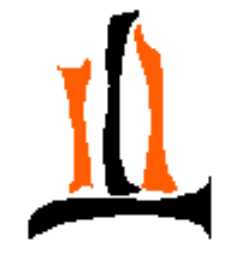

São Paulo 2004
Copyright 2004, SBGf - Sociedade Brasileira de Geofísica

Este texto foi preparado para a apresentação no I Simpósio de Geofísica da Sociedade Brasileira de Geofísica, São Paulo, 26-28 de setembro de 2004. Seu conteúdo foi revisado pela Comissão Tecno-científica do I SR-SBGf mas não necessariamente representa a opinião da SBGf ou de seus associados. E proibida a reprodução total ou parcial deste material para propósitos comerciais sem prévia autorização da SBGf.

\section{Resumo:}

O Laboratório de Desenvolvimento de Sensores Magnéticos do Observatório Nacional (LDSM/ON), desde 1998, desenvolve e constrói sensores e magnetômetros de princípios diversos, especialmente do tipo fluxgate. No núcleo sensor, temos ênfase no desenvolvimento de novos materiais adaptando-os e otimizando suas propriedades magnéticas. Este trabalho apresenta as etapas seguidas no desenvolvimento de um variômetro fluxgate construído para registrar variações, de baixa freqüência, do campo geomagnético, em observatórios magnéticos.

O sistema sensor é composto de três núcleos, de geometria toroidal, montados ortogonalmente $(X, Y, Z)$. Neste instrumento o sinal é processado analogicamente e na etapa final um conversor $A / D$ transforma-o em digital.

\section{Introdução: Teoria fluxgate}

A ação fluxgate é baseada na variação temporal da permeabilidade relativa do núcleo e os parâmetros: material e geometria, são determinantes na escolha de um sensor fluxgate.

O sensor fluxgate consiste basicamente de um núcleo construído com material ferromagnético de alta permeabilidade e envolto por dois sistemas de bobinas, uma responsável pela excitação e a outra para detecção o sinal induzido que traz a informação do campo externo. Alinhando o eixo longitudinal do sensor com o campo magnético externo, e excitando o núcleo com um sinal de corrente periódico com intensidade suficiente para saturar o núcleo, este será magnetizado alternadamente com o aparecimento de um fluxo magnético $(\Phi=$ B.A) no seu interior, proporcional à indução e a área da seção transversal do núcleo. Nestas condições a permeabilidade $(\mu)$ o material do núcleo sofrerá alteração acompanhando a variação do fluxo magnético. Como conseqüência um sinal de tensão $V$ será induzido nas $n$ espiras da bobina sensora. Considerando ainda outros fatores determinantes neste processo de magnetização, como o fator de desmagnetização (D) e a permeabilidade relativa do material $\left(\mu_{r}\right)$, tem-se a equação fundamental do sensor fluxgate:

$$
\mathrm{V}_{\mathrm{sec}}=\mathrm{n}_{\mathrm{s}} \mathrm{AB}_{\mathrm{ex}}(1-\mathrm{D}) \frac{\left(\mathrm{d} \mu_{\mathrm{r}} / \mathrm{dt}\right)}{\left[1+\mathrm{D}\left(\mu_{\mathrm{r}}-1\right)\right]^{2}}
$$

\section{Elemento sensor}

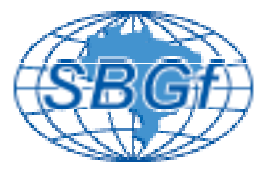

O sensor utiliza como núcleo uma liga magnética amorfa, de base cobalto e de composição $\mathrm{Co}_{67.5} \mathrm{Fe}_{3.5} \mathrm{Si}_{17.4} \mathrm{~B}_{11.6}$. Este material foi obtido pelo processo de "melt-spinner", em roda de ferro e atmosfera de argônio. A fita possui largura de $1.2 \mathrm{~mm}$, espessura de $0.002 \mathrm{~mm}$ e temperatura de Curie de $226^{\circ} \mathrm{C}$. Para otimizar as propriedades magnéticas, o material foi submetido a tratamento termomecânico adequado, que girou e reduziu a sua força coersiva. O sistema foi construído utilizando materiais selecionados visando homogeneizar seus coeficientes de dilatação térmica, reduzindo assim os efeitos das tensões internas, a serem provocadas quando o equipamento estiver funcionando sujeito a variações térmicas ambientais, reduzindo assim o nível de ruídos. O conjunto sensor é formado por três núcleos, de geometria toroidal, orientados em perpendicular.

\section{Alimentação/excitação}

A alimentação do circuito é conseguida através de uma fonte simétrica de 5 Volts contínuos, gerados à partir da rede AC normal, de 110 ou 220 Volts. Para garantir funcionamento correto dos circuitos, sem distorções, a estabilidade da alimentação é fundamental. Dessa maneira foram utilizados reguladores de tensão 7905 e 7805. Também foram utilizados capacitores de $100 \mathrm{nF}$, em paralelo com a alimentação dos circuitos integrados. Esta técnica proporciona uma redução no nível de ruídos da linha de transmissão. Optou-se ainda pelo uso de transistores em paralelo com os reguladores, de forma que a corrente seja limitada dentro de uma faixa fixa e pré-determinada, enquanto o restante é suprido pela malha dos transistores que pela configuração da a montagem funciona como um dissipador de calor.

O circuito é controlado por um oscilador de $8 \mathrm{MHz}$ que origina um sinal, de referência, com $31.250 \mathrm{KHz}$ de onda quadrada. Através de um divisor de freqüências, o sinal em dois, sendo um a ser usado como referencia e o outro que irá alimentar o núcleo do sensor através de uma excitação periódica de corrente e com forma de onda quadrada. Este sinal tem freqüência de $15.625 \mathrm{KHz}$ e intensidade $300 \mathrm{mApp}$. O sinal de saída, induzido na bobina sensora, é filtrado para eliminar todas os harmônicos pares, e resíduos de impares, permitindo apenas a passagem do segundo harmônico da excitação. Este sinal é então pré-amplificado, com a fase ajustada para ser comparada com o sinal de referência (puro da excitação). Depois de integrado, parte do sinal vai para uma bobina de compensação de campo que realimenta o sensor, fazendo com que o sistema opere em uma malha fechada, como um detetor de nulos. O sistema é todo chaveado permitindo analisar o circuito, como um todo,

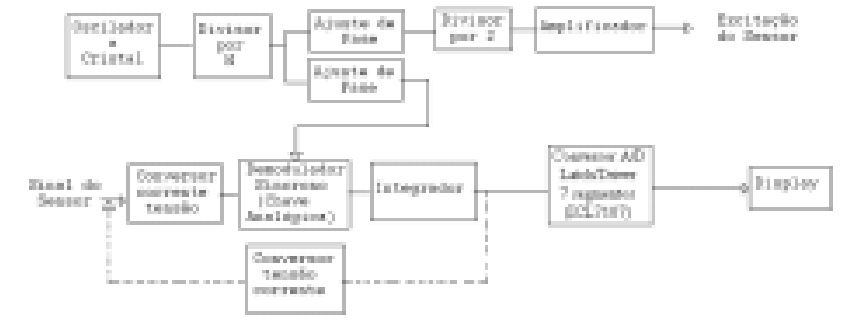




\section{Figura 1 - Diagrama em blocos do circuito}

\section{Detecção síncrona}

O demodulador tem como referência à freqüência de $31.250 \mathrm{KHz}$ gerada pelo oscilador. Este circuito, recebe o sinal amplificado do $2^{a}$ Harmônico e fornece na saída (emissor) amostras do semiciclo positivo e negativo deste sinal, conforme o sentido do campo externo gerador do sinal de 2a Harmônica. Estes pulsos são integrados pela rede $\mathrm{RC}$ tendo-se na saída um sinal $\mathrm{DC}$ de baixíssimo ripple, com intensidade proporcional à do campo externo e sinal negativo ou positivo, conforme o sentido do campo.

Amplificação dc - Em malha aberta este estágio fornece na saída uma tensão DC proporcional ao campo externo a ser medido. Em malha fechada, compensa a perda de sinal pela carga na bobina sensora causada pelos resistores de realimentação. Para ajustar o offset, foi fechada a entrada do ultimo estágio para a terra e ajustado o trimpot do amplificador para 0 Volts, na saída.

\section{Integrador}

Este estágio, em conjunto com a realimentação negativa aplicada à bobina sensora, fornece corrente suficiente para anular a saída do sensor e conseqüentemente do amplificador DC, formando um "detector de nulo". Considerando que um sistema operando em malha fechada é intrinsecamente mais estável que o mesmo sistema não realimentado. Uma vantagem adicional à estabilidade dos circuitos realimentados é o fato de que a linearidade decresce e o coeficiente de temperatura aumenta com o aumento do campo aplicado ao sensor. Procurou-se evitar também um substancial desvio de fase do sinal gerado no secundário com a amplitude. Em operação sem feedback, o coeficiente de temperatura do sensor é uma função da resistência do secundário. Com realimentação, a influência da resistência do secundário é minimizada pois seu valor é muito pequeno em comparação com 0 valor da resistência total de realimentação. O cancelamento do campo no interior da bobina não pode ser feito instantaneamente sendo dependente do tempo de integração e do ganho. Quanto maior o tempo de integração mais lenta a resposta. Quanto maior o ganho, mais rápida a integração. O ganho e a constante de tempo são balanceados para uma resposta adequada.

A bobina sensora é sintonizada para realçar o nível do $2^{\text {a }}$ Harmônico gerado pelo fluxgate, atenua as outras componentes e ruído. O capacitor ressonante ficou instalado junto à bobina sensora.

\section{Amplificação DC}

Em malha aberta este estágio fornece na saída uma tensão DC proporcional ao campo externo a ser medido. Em malha fechada, compensa a perda de sinal pela carga na bobina sensora causada pelos resistores de realimentação. Para ajustar o offset, foi fechada a entrada do ultimo estágio para a terra e foi ajustado o trimpot do amplificador para 0 Volts, na saída. $O$ instrumento responde à campos de pequena freqüência , até $0.5 \mathrm{~Hz}$, com re solução de $1.0 \mathrm{nT}$.

\section{Calibração e avaliação}

A avaliação de ruídos foi feita com o sistema sensor inicialmente dentro de uma blindagem magnética, cilíndrica, com cinco camadas concêntricas. Nesta foi considerada uma análise do sinal, por FFT, o que permitiu verificar o baixo ruídos do instrumento.

Para assegurar a orientação, individual, dos sensores foram realizadas uma série de medições, utilizando uma bobina, triaxial, quadrada, de Helmholtz que permitiu o comportamento dos sensores na presença de variações de campos da ordem de nT.

As respostas dos filtros usados no circuito foram determinadas por "software" fornecendo as curvas como a da figura 2 que mostra a resposta obtida por um filtro passa-baixa ativo de segunda ordem, com freqüência de corte (3 dB) em $0.23 \mathrm{~Hz}$, na saída do demodulador.

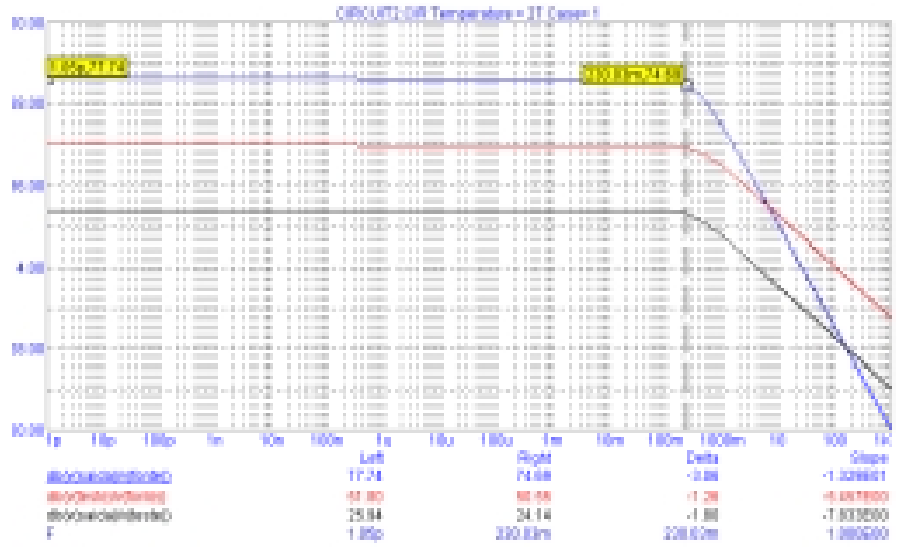

Figura 2 - Resposta, em freqüência, do filtro.

\section{Discussão e Conclusões:}

Nos testes de bancada a que foi submetido 0 equipamento aqui descrito, mostrou resultados que responde de maneira satisfatória aos propósitos a que se destina. Faz-se necessário realizar avaliações sobre seu desempenho no campo submetido a longo período de funcionamento, o que deve ser realizado em breve. Entretanto estes resultados já são bastante animadores considerando que o desenvolvimento e construção de variômetros, do tipo fluxgate, é de grande importância na atualização e modernização dos instrumentos de observatórios magnéticos.

\section{Agradecimentos}

Queremos agradecer a FAPERJ pelo apoio neste projeto e pelo suporte na concessão de bolsa (A.T.) para um de nós (M.B.).

\section{Referências}

Benyosef, L.C.C., Teodosio, J.R., Taranichev, V.E. \& Jalnin, B.V. 1995. Improvements on CoFeSiB Amorphous Ribbon for Fluxgate Sensor Cores - Scripta 
Metallurgica et Materialia, Pergamon-Elsevier Science Volume: 33 (9) - Págs. 1451-1454.

Benyosef, L. C. C. \& Novak, M. A. 1996. Magnetômetros de Núcleos Saturados, Revista de Instrumentação e de Física Aplicada, Sociedade Brasileira de Física.Vol. 11, Núm. 3, págs. 131-137.

Benyosef, L.C.C.; Teodósio, J.R.; Taranichev, V.E.; Zalnin, B.V. \& Nemova, O. Yu. 1996. Effect of Stress on the Magnetic Properties of Amorphous Ribbons for Fluxgate Magnetometers. Journal of Advanced Materials, Num. 3 (2) pags. 140-143

Benyosef, L.C.C. 1998. Development of Low Noise Fluxgate Sensors, Revista Geofísica, Instituto Panamericano de Geografia e História/IPGH - N. 48, 111-120

Cadorin, E. A.; Benyosef, L.C.C. \& Canázio, P.P.L.M. 2000. Magnetômetro de Núcleo Magnético Saturado, para Registro de Campos Magnéticos de Três Componentes $(X, Y, Z)$. Revista Militar de Ciência e Tecnologia, Vol. XVII. 42-48

Nielsen, O.V. Petersen et al (06 co-authors), 1995. Development, Construction and Analysis of the "Orsted" fluxgate magnetometer Meas. Sci. Technol. 6 pags. 1099-1115.

Primdhal, F., Petersen, J.R, Olin, C. and Harbo A.K. 1989. The short-circuited fluxgate output current, J. Phys. E.: Sci. Instrum. 22: 349-354. 


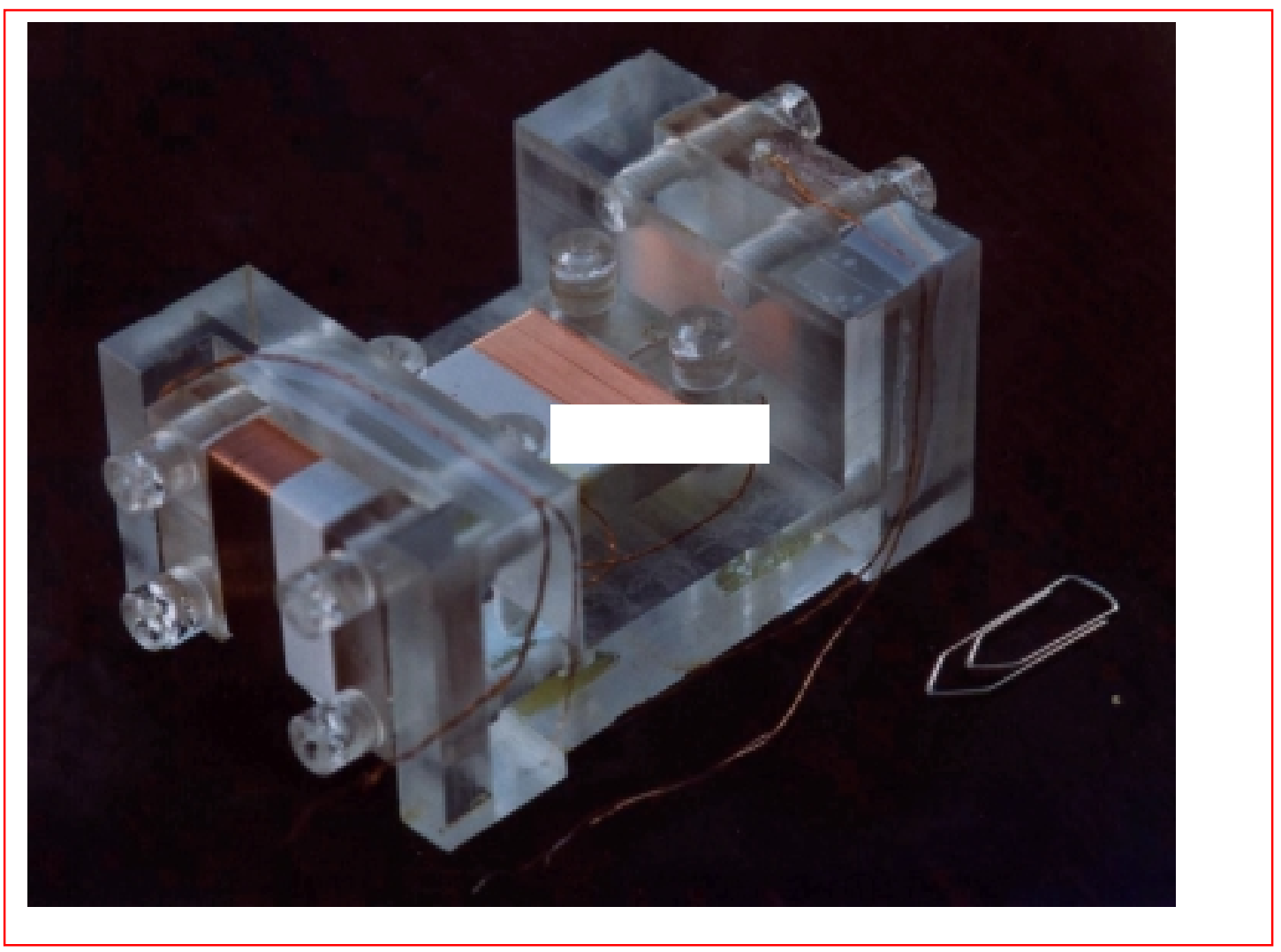

Finı ıra 4 - . Senconr flıxnate ıtilizadn nn variômetrn 